\title{
Estimación de curvas de carga en transformadores eléctricos mediante redes neuronales
}

\author{
Xiomara González-Ramírez*, Ángela M. Perea-Meneses*, \\ Gladys N. Caicedo*\$, Ferley Castro* \\ * Grupo de Investigación en Alta Tensión, Escuela de Ingeniería Eléctrica y Electrónica, \\ Universidad del Valle, Cali, Colombia \\ §e-mail: glacadel@univalle.edu.co
}

(Recibido: Junio 12 de 2008 - Aceptado: Noviembre 12 de 2008)

\section{Resumen}

Las curvas de carga en transformadores eléctricos se emplean para cuantificar las pérdidas en sistemas de distribución de energía, para establecer tarifas de cobro que varíen según la hora del día en que ocurre el consumo y para optimizar el uso de los transformadores. En este artículo se presenta un método para estimar las curvas de carga en transformadores de distribución. A partir de mediciones realizadas en una muestra de transformadores y de la información disponible en la base de datos comercial de una empresa de distribución de energía eléctrica, se entrenó una red neuronal por medio de una herramienta computacional basada en MATLAB. El método de estimación de las curvas de carga se implementó en el sistema de distribución de energía eléctrica de la ciudad de Buenaventura, Colombia. El aprendizaje de la red neuronal y el método para la estimación de las curvas de carga se validaron a través de su aplicación a un conjunto de transformadores distintos a los utilizados para el entrenamiento de la red neuronal, obteniéndose una precisión mayor al $90 \%$ en la estimación de las curvas de carga. En comparación con métodos tradicionales, el método presentado en este trabajo permite estimar la curva de carga de cualquier transformador de distribución de un modo más económico y preciso.

Palabras clave: Redes neuronales artificiales, Curva de carga, Consumo de electricidad, Transformador de distribución, Sistema de distribución.

\section{Estimation of load curves in electrical transformers by means of neural networks}

\begin{abstract}
Load curves in electrical transformers are employed to quantify losses in power distribution systems, to establish power utility rates that vary according to the hour of the day in which energy consumption occurs, and to optimize the use of transformers. This paper presents a method to estimate load curves in distribution transformers. From measurements made on a sample of transformers and the available information from the commercial database of an electrical power distribution company, a neural network was trained by means of a MATLAB-based computational tool. The load curve estimation method was implemented in the power distribution system of the city of Buenaventura, Colombia. The neural network learning and the load-curve estimation method were validated through their application to a set of transformers different from those used for training the neural network, obtaining an accuracy higher than $90 \%$ for the load curve estimation. As compared to traditional methods, the method presented in this work allows to estimate the load curve of any distribution transformer in a more economical and accurate way.
\end{abstract}

Keywords: Artificial neural networks, Load curve, Electrical power consumption, Distribution transformer, Distribution system. 


\section{Introducción}

Conocer la curva de carga en un transformador de distribución es importante en la operación y gestión de un sistema de distribución, ya que esta información se utiliza en estudios de cuantificación de pérdidas, definición de ofertas en la bolsa de energía, establecimiento de tarifas que varíen de acuerdo con la hora del día en que ocurre el consumo, reducción del pico de la demanda (gestión de carga), mejoramiento de la operación del sistema mediante el control en tiempo real y optimización de la utilización de los transformadores (Arias \& Bahamón, 2003).

Para estimar o predecir la demanda en un transformador de distribución se debe tener una metodología apropiada a las condiciones técnicas de cada sistema de distribución. Esta investigación utilizó el conjunto de variables que más influyen sobre la forma y la amplitud de la curva de la demanda en los transformadores de distribución. Estas variables, presentadas por (Arias \& Bahamón, 2003), son las siguientes: estrato socioeconómico, día de la semana, hora del día, porcentaje del tipo de usuarios conectados (residencial, comercial e industrial) y energía consumida.

La complejidad del problema de estimación de la curva de cualquier transformador de distribución se debe a los siguientes factores:

- Gran cantidad y variedad de usuarios de transformadores en un sistema de distribución.

- Definición y medición de las variables de mayor incidencia sobre la precisión.

- Gran volumen de información a procesar.

Es decir, la estimación de la carga involucra muchas variables a medir y procesar, lo cual implica un gran costo operativo, sí se realizan mediciones en cada transformador.

La estimación de la curva de carga en transformadores de distribución se ha hecho tradicionalmente por diferentes métodos, los cuales tratan de estimar con buena precisión la curva en cualquier transformador, considerando las variables más importantes involucradas en el problema. Dentro de los métodos para obtener la curva de carga en transformadores de distribución, se encuentran los métodos de medición, de agregación de curvas, de lógica difusa y de redes neuronales artificiales.

En los métodos de obtención de estas curvas por medición y agregación (Jardini et al., 1996; Ahn, 1997; Jardini et al., 2000; Arias \& Bahamón, 2003; Gallego et al., 2007), la obtención de la curva de carga para cada transformador de distribución se realiza a través de mediciones directas en el lado de baja, donde se censan las variables eléctricas todos los días de la semana en intervalos de 1,5 o $15 \mathrm{~min}$, durante 15 días, aproximadamente. Esta información es procesada mediante herramientas estadísticas que permiten obtener una curva típica de carga para el transformador medido. El método por medición permite obtener la curva de carga del transformador con una alta precisión, pero no permite estimar la curva en otros transformadores del sistema; en contraste, el método de agregación de curvas de carga sí permite obtener la curva de carga en otros transformadores del sistema.

El método de obtención de las curvas mediante lógica difusa (Konjic et al., 2004; Pérez-Cisneros \& Vernon, 2005) permite rapidez en el procesamiento y precisión en los resultados. Sin embargo, el principal inconveniente de este método radica en la dificultad de elegir una correcta función de pertenencia para los conjuntos difusos aplicados al problema de estimación. Este método también permite estimar la curva de carga en otros transformadores del sistema.

Por último, se encuentra el método de obtención de las curvas mediante redes neuronales artificiales (RNA) (Ahn, 1997; Mallo-González, 2004; Villanueva-Espinoza, 2002), el cual no requiere el desarrollo de relaciones matemáticas complejas para correlacionar los datos, lo que frecuentemente permite reducir el trabajo de programación. El método de RNA presenta gran capacidad de adaptación ante problemas no lineales y un buen desempeño en el manejo de gran cantidad de información, lo que permite procesar todas las mediciones de la muestra de transformadores seleccionados en el sistema de 
distribución. Para resolver un problema determinado mediante una RNA, se debe realizar un proceso de entrenamiento a partir de un conjunto de datos tomados de varias curvas reales, que permitan a la red neuronal extraer las reglas básicas de su comportamiento.

Después de procesar los datos de la muestra, la red neuronal artificial (RNA) encuentra sus patrones característicos, los cuales pueden ser proyectados sobre los demás transformadores de distribución; es decir, la RNA permite estimar la curva de carga en otros transformadores del sistema. De acuerdo a la literatura consultada, la precisión de la estimación mediante el método de RNA es mejor que con los métodos anteriores. Además, la posibilidad de hacer la estimación en otros transformadores disminuye los costos económicos, ya que no es necesario realizar mediciones en todos los transformadores del sistema. La gran fortaleza de esta metodología es que permite estimar la curva de carga de cualquier transformador de un sistema de distribución. Si se cambian las características de los usuarios del transformador, la red se entrena de nuevo para obtener nuevos patrones.

\section{Metodología}

El problema de estimación de la demanda en un transformador de distribución consiste en obtener una metodología apropiada a las condiciones técnicas y culturales de cada sistema, de tal forma que se involucren las variables más significativas y los procedimientos estadísticos adecuados que permitan obtener una curva de carga exacta o que se aproxime a la realidad.
La toma de datos y la medición en un sistema de distribución son procesos complejos y costosos, ya que se requiere medir diferentes variables. Además, la mayoría de los métodos requieren el uso de procesos estadísticos donde se debe procesar un gran volumen de información, ocasionando en las herramientas computacionales un retardo en el manejo del proceso debido a que se requiere procesar la información para cada transformador del sistema. Por esto, se busca un método que pueda procesar una gran cantidad de información en forma rápida, precisa y efectiva, con el fin de obtener las curvas de carga. Por estas razones, en este trabajo se aplicó la técnica de redes neuronales artificiales.

Las curvas de carga de un transformador de distribución se miden generalmente en el lado de baja del transformador. Estas curvas representan la variación de la potencia activa $P(t)$ o reactiva $Q(t)$ durante el día (Mallo-González, 2004).

Las curvas de carga cambian de amplitud y forma de acuerdo a las siguientes variables:

- Porcentaje de tipos de usuarios conectados al transformador.

- Día de la semana.

- Energía consumida en un intervalo de tiempo.

• Estrato socioeconómico.

En la Figura 1 se ilustra la influencia de cada una de estas variables sobre la curva de carga.

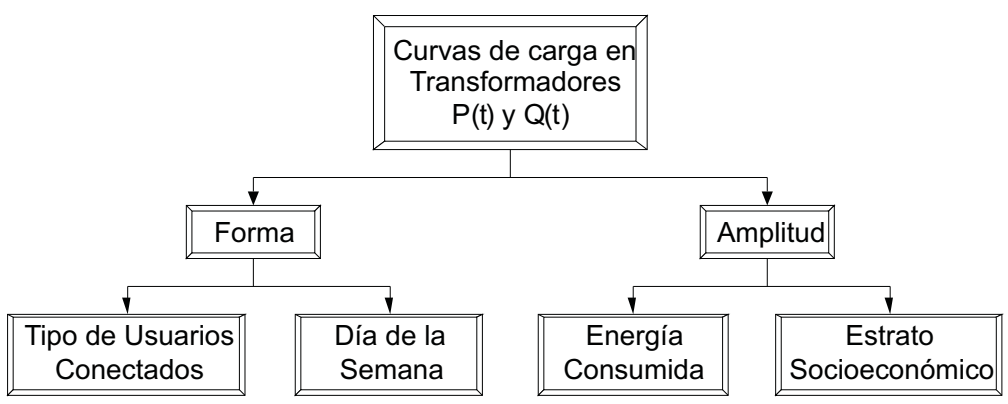

Figura 1. Variables que afectan las características de las curvas de carga. 
La metodología desarrollada en esta investigación se resume en 7 pasos:

\subsection{Toma de datos en la muestra de transformadores}

En la muestra de transformadores seleccionados, se definen las variables eléctricas a medir que más influyen en la precisión de los resultados.

Se consideraron los siguientes criterios para seleccionar la muestra de transformadores y las variables a medir:

- Transformadores de todos los circuitos de distribución.

- Transformadores con diferentes tipos de carga.

- Variables de medición: potencia activa $P(t)$, potencia reactiva $Q(t)$, energía activa consumida $\mathrm{kW}-\mathrm{h}(t)$, energía reactiva consumida $\mathrm{kVAr}-\mathrm{h}(t)$, voltaje $V(t)$, intensidad de corriente $I(t)$, factor de potencia $f_{p}(t)$. Cada variable se midió cada $15 \mathrm{~min}$ durante los 7 días de la semana.

Las mediciones se realizaron sobre una muestra de 50 transformadores de distribución del municipio de Buenaventura, Colombia.

\subsection{Procesamiento de la información}

Se utilizan herramientas estadísticas para obtener las curvas típicas (representativas) de la carga para cada muestra.

Para obtener las curvas típicas de carga de cada transformador de la muestra, se utilizó el paquete estadístico S-Plus. A cada una de las curvas medidas en el transformador de la muestra se le hizo el siguiente procesamiento:

- Cambio de valores reales a valores por unidad (p.u.)

- Procesamiento estadístico para obtener una curva suavizada.

- Obtención de la curva típica unificada de lunes a viernes para cada transformador mediante la técnica de suavización Kernel, la cual consiste en obtener los promedios ponderados de todos los datos de las curvas de carga para un tiempo determinado, como se ilustra en la Figura 2.

\subsection{Clasificación de las curvas}

Se hace una clasificación de las curvas de acuerdo a sus características particulares de consumo. Esta clasificación permite definir un grupo de curvas para un conjunto de transformadores con características de entrada similares. Esta clasificación preliminar mejora la calidad del entrenamiento de la red y, por lo tanto, aumenta la precisión en la estimación. Por cada grupo de curvas clasificadas, la red neuronal define un patrón, el cual representa el comportamiento característico para las curvas de ese grupo de transformadores.

Esta clasificación de transformadores con características similares, es lo que permite entrenar la red y obtener una curva patrón para esta muestra. Aunque la clasificación se realizó de forma manual en esta investigación, existen algunas herramientas computacionales que permiten hacer esta clasificación automáticamente.

\subsection{Selección e implementación de la red}

Con las curvas típicas de carga y las variables de entrada seleccionadas que más inciden en su forma y amplitud, se estructuró la red neuronal, la cual se implementó en MATLAB V 7.2.

La red seleccionada para la solución a este problema es la red perceptron multicapa (MLP) y se empleó el algoritmo de retropropagación, el cual permite resolver problemas no lineales, y minimizar el error de las capas ocultas reajustando los pesos de conexión de cada neurona (Villanueva-Espinoza, 2002). La estructura general de la red utilizada para este problema, se ilustra en la Figura 3.

La implementación de la estructura de la red neuronal se realizó con la correspondiente caja de herramientas de MATLAB, la cual requiere de un vector de entradas, de un vector objetivo y de parámetros de la red neuronal tales como el tipo de red, la función de entrenamiento, el número de 


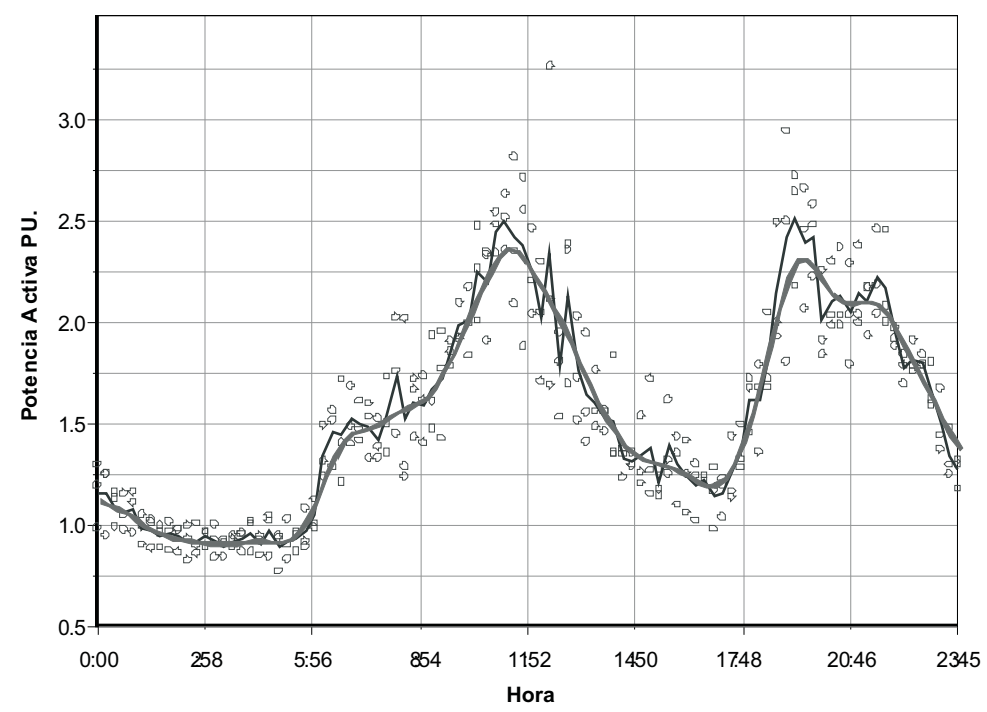

Figura 2. Curvas típicas de las muestras suavizadas mediante la herramienta estadística S-Plus.

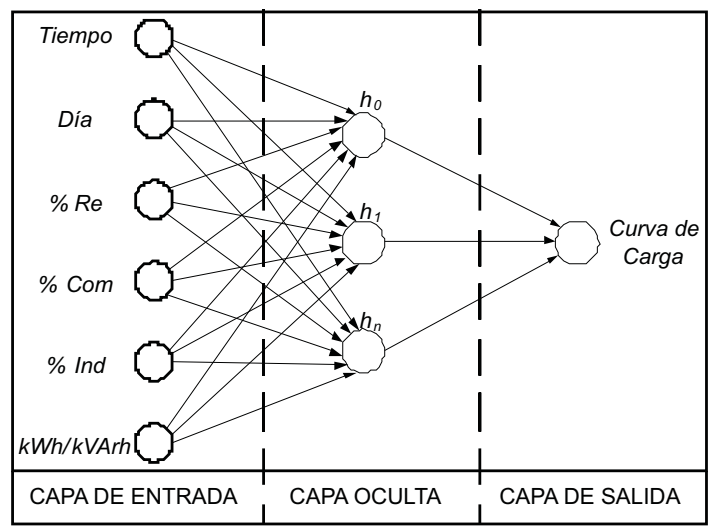

Figura 3. Estructura general de la RNA utilizada.

capas, el número de neuronas y el número de iteraciones.

En la Figura 4 se presenta la estructura funcional de la herramienta computacional desarrollada. Se ilustran las funciones de entrenamiento, de validación y de estimación de las curvas de carga mediante RNA.

\subsection{Entrenamiento de la red neuronal}

A partir de las características comunes de las curvas de la muestra, se realiza el entrenamiento de la red neuronal y se encuentran los patrones de aprendizaje.
Para ese entrenamiento, en esta investigación se utilizó el $70 \%$ de las curvas típicas de los transformadores de las muestras. En la Figura 5 se presenta el diagrama funcional que permite realizar el entrenamiento de la red neuronal.

\subsubsection{Entrada de matrices}

Como se mencionó, el entrenamiento de la red se realiza con las siguientes variables: $P(t)$ y $Q(t)$ (suavizadas y en valores por unidad), día de la semana, porcentaje del tipo de usuarios conectados (residencial, comercial e industrial) y energía consumida ( $\mathrm{kW}-\mathrm{h} / \mathrm{kVAr}-\mathrm{h})$ medida cada $15 \mathrm{~min}$. 


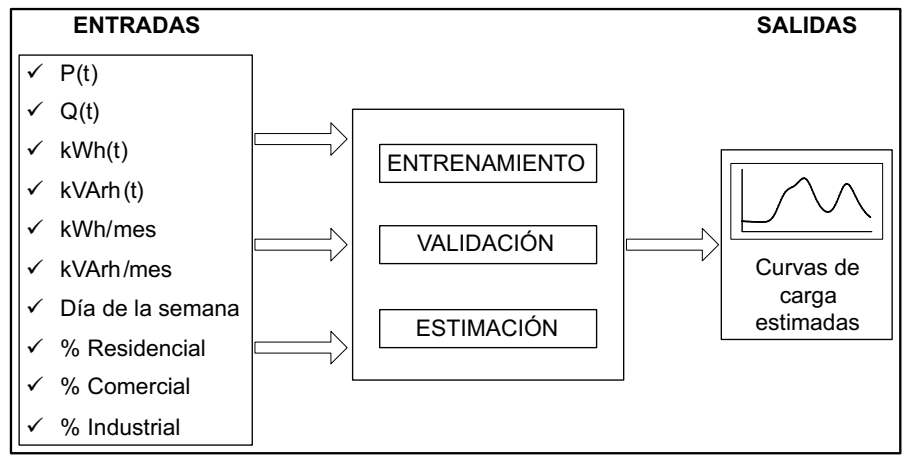

Figura 4. Estructura funcional de la herramienta computacional desarrollada utilizando RNA.

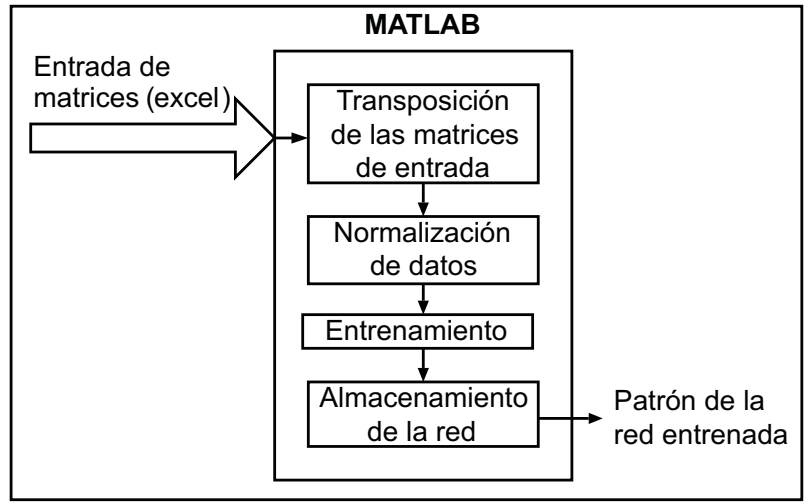

Figura 5. Diagrama de la etapa de entrenamiento.

Estas variables se organizaron en forma de matrices y se almacenaron en Excel, debido a que es un programa de fácil manejo para la importación de datos en MATLAB. Como se muestra en la Tabla 1, en cada columna se encuentran las variables o datos de entrada de la red y en cada fila el valor correspondiente a cada intervalo de tiempo.

\subsubsection{Transposición de las matrices de entrada}

Las variables de entrada se leen por filas en MATLAB. Por esto, se realizó la transposición de las matrices de los datos de entrada, es decir, se cambiaron filas por columnas para que la red lograra leer los datos correctamente.

\subsubsection{Normalización de datos}

Esta normalización requerida por MATLAB, consiste en modificar la escala de los datos de entrada atendiendo a su valor mínimo y máximo, linealmente escaladas entre -1 y 1 . De esta forma, se obtiene un conjunto de datos más homogéneo, lo cual permite que el aprendizaje de la red sea más fácil y rápido.

\subsubsection{Variables del entrenamiento}

La red implementada consta de tres capas: una de entrada, una oculta y una de salida. La capa de entrada cuenta con 6 neuronas que representan las variables consideradas en las muestras. La capa de salida cuenta con una neurona, que indica el valor de la potencia eléctrica $(P \circ Q)$ asociada a cada vector de entrada presentado a la red. La capa oculta tiene un número variable de neuronas, las cuales se determinan por ensayo y error en cada entrenamiento. Es decir, el número de neuronas se ajusta a medida que se realiza el entrenamiento con el fin de encontrar un mínimo error. Es importante tener en cuenta que no se deben 
Tabla 1. Organización de datos en forma de matriz.

\begin{tabular}{|c|c|c|c|c|c|c|}
\hline Tiempo & Día & $\%$ Re & $\%$ Co & \%In & kW-h & Potencia activa \\
\hline $0: 00$ & 1 & 100 & 0 & 0 & 4.6044 & 0.3577 \\
\hline $0: 15$ & 1 & 100 & 0 & 0 & 4.5019 & 0.3497 \\
\hline $0: 30$ & 1 & 100 & 0 & 0 & 4.4064 & 0.3423 \\
\hline $0: 45$ & 1 & 100 & 0 & 0 & 4.3221 & 0.3358 \\
\hline $1: 00$ & 1 & 100 & 0 & 0 & 4.2413 & 0.3295 \\
\hline $1: 15$ & 1 & 100 & 0 & 0 & 4.1503 & 0.3224 \\
\hline
\end{tabular}

* El día de la semana se representa por números:

$1=$ Domingo

$2=$ Lunes - Viernes

$3=$ Sábados
$\%$ Re: porcentaje de usuarios residenciales $\%$ Co: porcentaje de usuarios comerciales $\%$ In: porcentaje de usuarios industriales

\subsection{Estimación de las curvas de carga}

Se realizó un ajuste en la variable de la energía para realizar la estimación con valores de energía mensual. La estimación de la curva de carga $P(t)$ o $Q(t)$ puede realizarse para cualquier transformador de distribución que tenga características de entrada similares a las utilizadas en el entrenamiento. Si el transformador tiene otras características, la red debe entrenarse de nuevo con el fin de encontrar el patrón característico para este tipo de transformador.

En esta investigación, la estimación se validó con el $30 \%$ de las muestras restantes que no fueron utilizadas en el entrenamiento.

Para estimar la curva de un transformador, se deben definir en la herramienta desarrollada el día de la semana y el tipo de curva $P(t)$ o $Q(t)$, y se debe cargar la red patrón entrenada para el tipo de curva determinada.

Además de la base de datos comercial de la empresa de energía, se debe obtener el porcentaje de tipo de usuarios conectados (residencial, comercial e industrial) y la energía activa o reactiva mensual consumida.
El procedimiento de validación de la red se ilustra en el esquema de la Figura 6. 


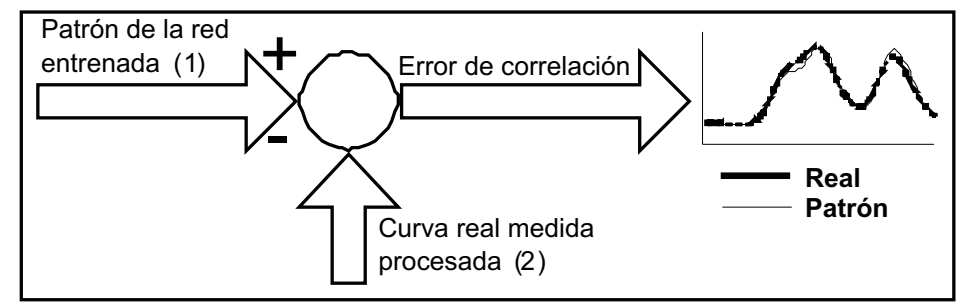

Figura 6. Esquema de validación de la red neuronal.

(1) Curva de carga patrón obtenida del entrenamiento, con el $70 \%$ de las curvas reales procesadas de la muestra.

(2) Curva real seleccionada del $30 \%$ restante de la muestra.

Tanto (1) como (2) deben estar en el mismo grupo de clasificación de curvas, que corresponde a

transformadores con características similares.

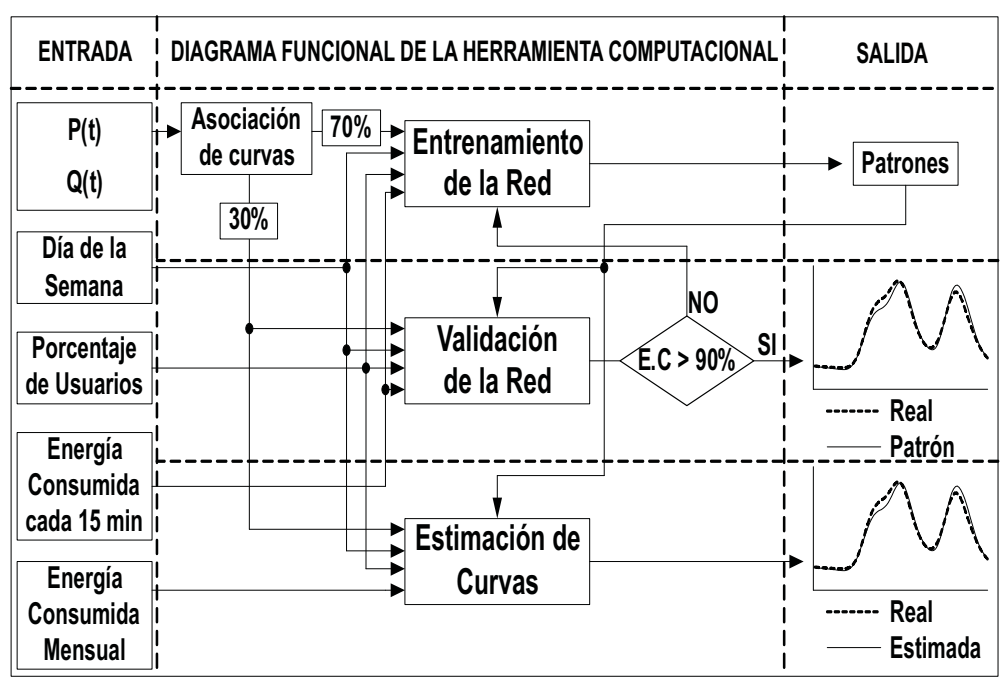

Figura 7. Diagrama funcional de la red neuronal implementada.

En la Figura 7 se presenta el diagrama funcional de la red neuronal implementada.

\section{Resultados}

Como primer resultado se presenta la herramienta computacional desarrollada en esta investigación, denominada ESCATD (estimación de la curva de carga en transformadores de distribución mediante redes neuronales). Esta herramienta se ilustra en la Figura 8, en la cual se aprecian las funciones de entrenamiento, validación y estimación.
Con base en los resultados del entrenamiento, se selecciona la mejor red entrenada (patrón) para utilizarla en la estimación.

La validación se realizó para dos casos:

\subsection{Validación del entrenamiento de la curva patrón}

En este caso se comparó la curva patrón entregada por la red, con una curva seleccionada del grupo del $70 \%$ de la muestra de transformadores. En la Figura 9 se aprecia la similitud entre ambas curvas, la cual se conserva para cada una de las curvas de grupo de la muestra. 


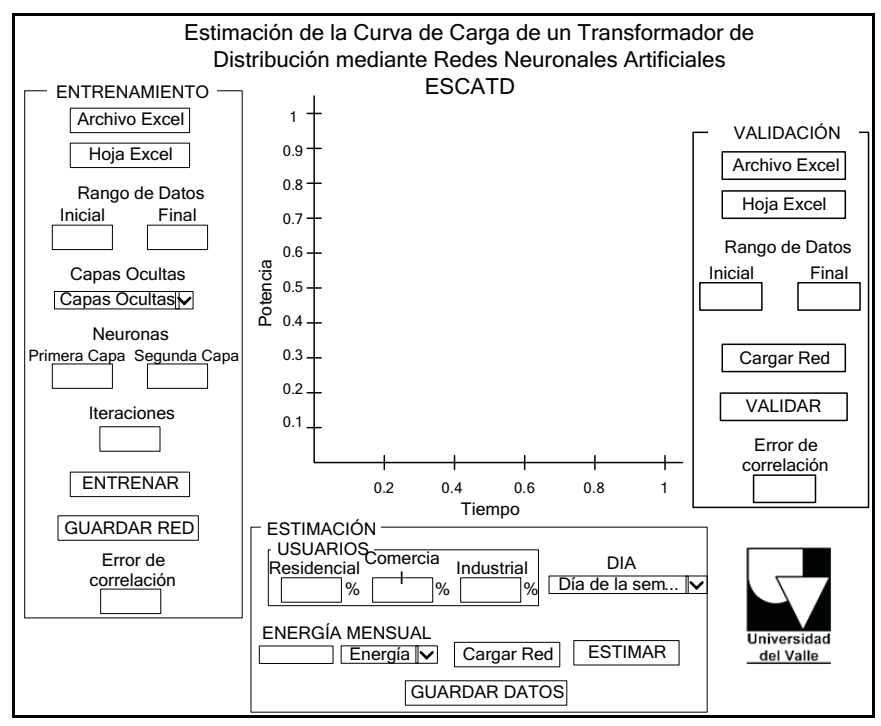

Figura 8. Interfaz del ESCATD.

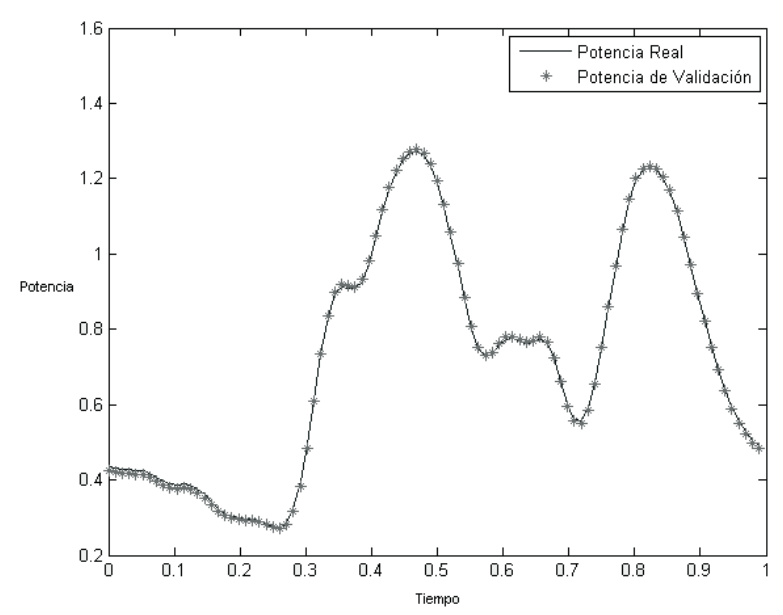

Figura 9. Validación del entrenamiento.

\subsection{Validación de la curva de carga estimada}

En este caso se comparó la curva patrón entrenada y curva validada (estimada), con cada curva de carga seleccionada del grupo del $30 \%$ restante de la muestra de transformadores. En la Figura $10 \mathrm{se}$ aprecia la validación de la estimación para una curva del grupo y se observa la similitud de ambas curvas.

En la Tabla 2 se presentan los resultados de la precisión de la curva estimada, con las demás curvas del grupo del $30 \%$ restante de la muestra.
Se observa en la Tabla 2 que la precisión para el $30 \%$ de las muestras restantes es aproximadamente igual a 1 , lo que indica una gran asociación entre la curva real y la curva validada por la red neuronal. Es decir, que la respuesta de la red entrenada es satisfactoria para realizar la estimación de curvas de cargas desconocidas.

Finalmente, en la Tabla 3 se presentan los resultados totales de la etapa de entrenamiento de la red neuronal y de la validación de las curvas estimadas. 
Tabla 2. Resultados de validación del entrenamiento.

\begin{tabular}{|c|c|c|c|}
\hline \multirow{2}{*}{ Muestra } & \multicolumn{3}{|c|}{ Precisión } \\
\cline { 2 - 4 } & Día (1) & Día (2) & Día (3) \\
\hline 4 & 0.99898 & 0.99922 & 0.99956 \\
\hline 7 & 0.99993 & 0.99992 & 0.99991 \\
\hline 9 & 0.99990 & 0.99981 & 0.99980 \\
\hline 12 & 0.99932 & 0.99895 & 0.99922 \\
\hline 15 & 0.99914 & 0.99917 & 0.99883 \\
\hline 16 & 0.99981 & 0.99979 & 0.99977 \\
\hline 18 & 0.99628 & 0.99538 & 0.99667 \\
\hline 19 & 0.99995 & 0.9999 & 0.99992 \\
\hline 27 & 0.99996 & 0.99989 & 0.99993 \\
\hline 28 & 0.99993 & 0.99991 & 0.99994 \\
\hline 31 & 0.99863 & 0.99847 & 0.99780 \\
\hline 32 & 0.99995 & 0.99993 & 0.99984 \\
\hline 33 & 0.99987 & 0.99974 & 0.99971 \\
\hline 36 & 0.99925 & 0.99926 & 0.99894 \\
\hline 50 & 0.99725 & 0.99992 & \multirow{2}{|c}{} \\
\hline & \multicolumn{3}{|c}{} \\
\hline
\end{tabular}

Cuando se requiere estimar la curva de carga para un transformador con características similares y que no pertenece a la muestra, se debe contar con el dato de energía mensual consumido, el cual se obtiene de la base de datos comercial (ya que para este transformador no se tiene ninguna medición).

En la Figura 11 se observa la influencia que tiene la variable de consumo de energía mensual del transformador sobre la precisión de la curva estimada. En este caso se utilizó un valor de energía mensual consumida mayor que el rango utilizado en la curva patrón entrenada y se observa que las curvas son similares en forma pero no en amplitud.
En la Figura 12 se utilizó un valor de energía mensual consumida menor que el rango utilizado en la curva patrón entrenada. Al igual que en la Figura 11, se observa que las curvas son similares en forma pero no en amplitud.

\section{Conclusiones}

Para estimar la curva de carga de un transformador con una buena precisión se deben considerar las siguientes variables: día de la semana, porcentaje de tipo de usuario y consumo mensual de energía.

La precisión obtenida para las 45 curvas estimadas, tanto para potencia activa y reactiva, estuvo por encima del $90 \%$. Esto significa que para obtener la curva de carga no es necesario realizar mediciones en todos los transformadores de distribución, sino que estas pueden ser estimadas con una buena precisión, lo cual implica una reducción de costos para las empresas del sector eléctrico que requieren las curvas de carga de diferentes transformadores para diversos estudios.

La herramienta computacional desarrollada en esta investigación es flexible, ya que seleccionando un grupo de curvas de transformadores con características similares, se puede entrenar la RNA y obtener nuevos patrones, los cuales permitirán estimar la curva de carga de otro tipo de transformador.

Las RNA son herramientas de tratamiento de información que pueden integrarse fácilmente en problemas de estimación. Como se observó en este caso, para la obtención de la curva de carga en transformadores de distribución, la función de estimación logra precisar la curva de carga a partir de los datos existentes de la base comercial de las empresas distribuidoras de energía.

Tabla 3. Resultados de entrenamiento y validación de la estimación.

\begin{tabular}{|lcccc|}
\hline \multicolumn{1}{|c}{ Etapa } & Curvas & Neuronas & Iteraciones & Precisión \\
\hline Entrenamiento & 105 & 24 & 200 & $>90 \%$ \\
\hline Validación & 45 & - & - & $>90 \%$ \\
\hline
\end{tabular}




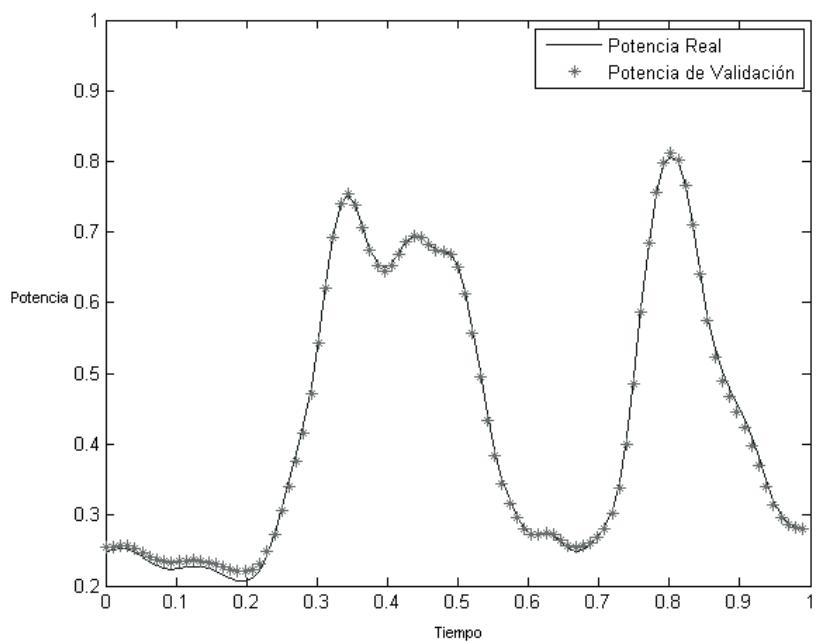

Figura 10. Validación de la estimación de la curva de carga de un transformador.

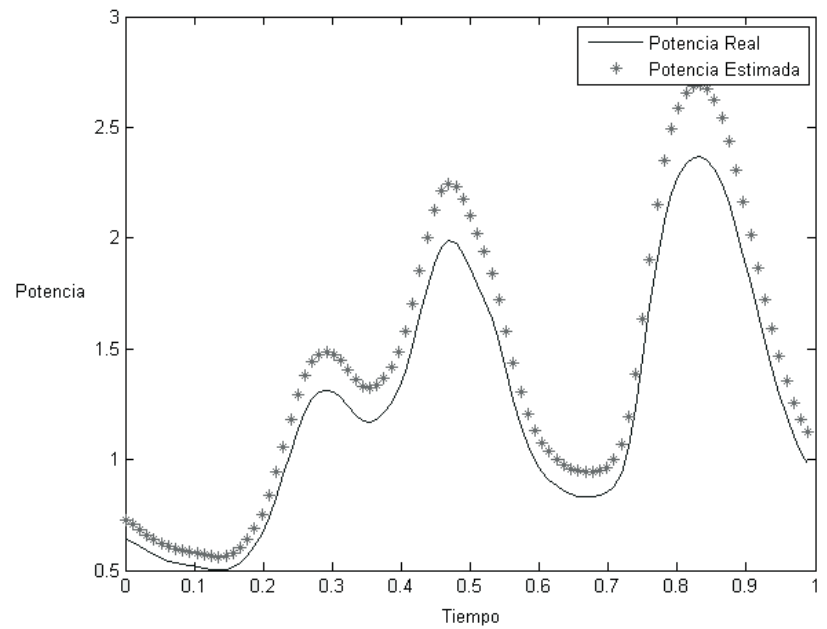

Figura 11. Curva de carga estimada con el valor de la energía mensual por encima del valor real.

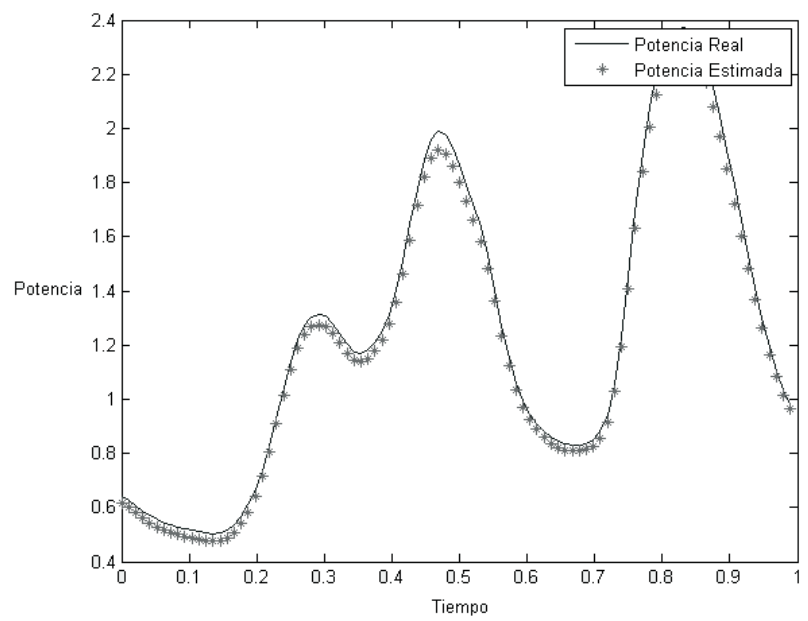

Figura 12. Curva estimada con el valor de la energía mensual por debajo del valor real. 


\section{Referencias bibliográficas}

Ahn, S. U. (1997). Metodologia para Seleção e Gerenciamento de Transformadores de Distribuição, aplicando Técnicas de Redes Neurais Artificiais. Tese de Doutorado, Departamento de Engenharia de Energia e Automação Elétricas, Escola Politécnica da Universidade de São Paulo, São Paulo, Brasil.

Arias, L. L., \& Bahamón, A. (2003). Una nueva metodología para la demanda en sistemas de distribución. Trabajo de grado, Escuela de Ingeniería Eléctrica y Electrónica, Universidad del Valle, Cali, Colombia.

Gallego, L. A., Francisquini, A., Gómez, O., \& Padilha A. (2007). Estimación de curvas de demanda de consumidores, transformadores de distribución y alimentadores primarios en sistemas de distribución. Scientia e Technica 13 (35), 107-112.

Jardini, J. A., Tahan, C. M. V., Ahn, S. U., \& Ferrari, E. L. (1996). Distribution transformer loading evaluation based on load profiles measurements. IEEE Transactions on Power Delivery 12 (4), 1766-1770.

Jardini, J. A., Tahan, C. M. V., Gouvea, M. R., Ahn, S. U., \& Figueiredo, F. M. (2000). Daily load profiles for residential, commercial and industrial low voltage consumers. IEEE Transactions on Power Delivery 15 (1), 375-380.
Konjic, T., Miranda, V., \& Kapetanovic, I. (2004). Prediction of $L V$ substation load curves with fuzzy inference systems. Eight International Conference on Probabilistic Methods Applied to Power Systems (PMAPS), Ames, Iowa, U.S.A., p. 129-134.

Mallo-González, C. (2004). Predicción de la demanda eléctrica horaria mediante redes neuronales artificiales. Revista Electrónica de Comunicaciones y Trabajos de ASEPUMA $5(1), 5-28$.

http://www.uv.es/asepuma/recta/n5.htm

Perez-Cisneros, M. A., \& Vernon, J. (2005). Sistemas de lógica difusa. In Control Systems Principles web page:

h t t p : / / w w w . c o n t r o l-s y s t e m s principles.co.uk/whitepapers/spanishwp/13fuzzy logicSP.pdf

Rodríguez-Ponce, H. U. (2004). Perceptrón multicapa para reconocimiento de objetos sobre planos. Tesis Profesional para la Licenciatura en Ingeniería en Electrónica y Comunicaciones, Departamento de Ingeniería Electrónica, Universidad de las Américas Puebla, Puebla, México.

http://catarina.udlap.mx/u_dl_a/tales/ documentos/lem/rodriguez_p_hu/

Villanueva-Espinoza, M. R. (2002). Las redes neuronales artificiales y su importancia como herramienta en la toma de decisiones. Trabajo de Investigación para la Licenciatura, Facultad de Ciencias Matemáticas, EAP de Investigación Operativa, Universidad Nacional Mayor de San Marcos, Lima, Perú.

http://sisbib.unmsm.edu.pe/bibVirtual/Tesis/ Basic/Villanueva_EM/Contenido.htm 